\title{
Two-plane focusing of high-space-charge sheet electron beams using periodically cusped magnetic fields
}

\author{
M. A. Basten ${ }^{\text {a) }}$ and J. H. Booske ${ }^{\text {b) }}$ \\ Department of Electrical and Computer Engineering, University of Wisconsin, Madison, Wisconsin 53706
}

(Received 18 September 1998; accepted for publication 26 January 1999)

\begin{abstract}
Numerical and theoretical analyses show that stable, two-plane focusing of finite width, elliptical cross section, sheet electron beams with high space charge (low voltage, high current density) can be accomplished using periodically cusped-magnetic (PCM) fields. Magnetic field strength requirements for focusing high-space-charge sheet beams are within technological capabilities of modern permanent magnet technology. Both an offset-pole PCM stack and a PCM stack combined with a periodic quadrupole magnet (PQM) edge array are shown to be effective for two-plane sheet beam confinement. The PCM-PQM hybrid configuration offers inherent advantages for independent adjustment of confinement fields to achieve beam matching (minimum ripple) in both transverse dimensions. The offset-pole configuration offers the advantage of open-side access for applications such as vacuum electronic microwave devices. It is also shown that PCM-focused sheet beam envelope stability obeys criteria equivalent to that previously identified for round-cross-section electron beams in periodic permanent magnet focusing. (C) 1999 American Institute of Physics.
\end{abstract} [S0021-8979(99)03809-8]

\section{INTRODUCTION}

The use of extended beam configurations, especially sheet electron beams, has the potential to greatly advance the state of the art for vacuum microwave devices. Particularly as the current trend towards the high-frequency millimeterwave band continues, where device dimensions tend to decrease with wavelength, there is a need to overcome limitations inherent in conventional round beam devices. Sheet electron beams have the advantage that the total amount of beam current in a single device, and therefore the total output power, may be increased without inordinate increases in the beam space-charge density. Therefore a sheet-beam microwave tube can largely avoid both direct current (dc) and alternating current (ac) defocusing problems due to beam space charge and subsequent efficiency loss. Similarly, the extended type of radio frequency (rf) interaction circuit compatible with sheet beams allows for increased total rf power capacity without electric breakdown.

The advantages of using sheet electron beams for the transport of high current through microwave circuits were appreciated at least as early as the mid-1950's. ${ }^{1-4}$ Soon thereafter experimental investigations of the transport of sheet beams in uniform axial magnetic fields showed the existence of an instability which led to beam kink and filamentation. ${ }^{5-7}$ It was recognized that the driving mechanism behind that instability was linked to the coupling of the radial motion of the beam, induced by space charge, with the focusing provided by the longitudinal magnetic field. ${ }^{6,8}$ In the later work, it was shown that the driving mechanism was indeed due to an $\mathbf{E} \times \mathbf{B}$ velocity shear across the top and bottom of the electron beam, ${ }^{9-11}$ where $\mathbf{E}$ is the transverse

${ }^{\text {a) }}$ Current address: Northrop Grumman Corporation, Rolling Meadows, IL.

${ }^{\text {b) }}$ Electronic mail: booske@engr.wisc.edu space-charge electric field and $\mathbf{B}$ is the longitudinal magnetic field. Now referred to as the diocotron instability, the phenomena largely discouraged the investigation of sheet electron beams in microwave devices until relatively recently.

Periodic focusing, rather than a uniform guide field, was proposed as a possible method to avoid filamentation. Early concepts investigated electrostatic periodic focusing of sheet beams, ${ }^{2,12}$ but Sturrock pointed out that magnetic periodic focusing had the potential to focus higher current beams for reasonable magnet field strengths. ${ }^{3}$ Although Sturrock considers beam "stability" in this work (and in a companion article on a general theory of sheet beams in periodic fields ${ }^{4}$ ), the stability criterion thus developed is actually a condition for effective single-particle transport of the beam through the focusing stack. Although later analyses, mainly based on the plasma fluid equations, ${ }^{11,13,14}$ have investigated the issue of sheet beams in uniform focusing under a variety of conditions, few investigations of sheet-beam stability in periodic fields have been undertaken.

One notable exception is recent work done at the University of Maryland as part of a sheet-beam free-electron laser experiment. ${ }^{15}$ This work investigated the stability of sheet beam transport through a $1 \mathrm{~cm}$, five-period, 800-1600 g planar wiggler array. The "sheet' beam actually begins as a planar multiple beamlet array produced by seventeen 1.0 $\mathrm{mm}$ holes drilled into an anode plate approximately $3.0 \mathrm{~mm}$ apart. However, by the end of the transport channel $(5 \mathrm{~cm})$, the beamlets merge into a continuous sheet approximately $3 \mathrm{~mm} \times 30 \mathrm{~mm}$ in extent. The beam used in this experiment was relativistic - 100 and $400 \mathrm{kV}$ beam voltages - and produced by a field-emission ("cold") cathode for short $(<100$ ns) pulses. About $26 \mathrm{~A}$ of current was present in the beam after scraping at the anode plate. Hence, the experimental parameters used in this study are in the relativistic (highvoltage), low-space-charge regime - a fact bolstered by the 
relatively good agreement of the actual beam transport with single-particle codes mentioned in the article.

As an indication of the increasing interest in and appreciation of sheet beams for use in microwave devices, a recent paper design study compares a sheet beam klystron to round beam klystrons at $11.4 \mathrm{GHz}$ for accelerator applications. ${ }^{16}$ Due to the high beam current, only moderate beam voltages $(400 \mathrm{kV})$ are necessary to provide the same output power as highly relativistic $(3000 \mathrm{kV})$ klystrons. In addition, an efficiency enhancement (by a factor of 2-4) is deemed possible through the reduced space charge in the sheet beam. Solenoidal focusing is envisioned for this device. This work was based on an earlier paper design study ${ }^{17}$ using a planar magnetic wiggler for beam focusing. Although these designs appear to provide marked improvement in the state of the art of high-power klystrons, the experimental demonstration has yet to be made and, furthermore, basic questions of sheetbeam stability and transport need to be addressed.

A particular type of focusing configuration-the offsetpole periodically cusped-magnet (PCM) array-has been identified which provides stable focusing in both transverse dimensions of the beam and the low transverse velocities compatible with linear beam microwave tubes. Recent articles have shown that space-charge-dominated, infinitely wide sheet electron beams can be stabilized in periodically varying magnetic field configurations based on the PCM array. ${ }^{18-20}$

This article describes the results of advanced theoretical and computational studies of the focusing and transport of sheet electron beams in planar PCM fields. The beam dynamics are examined using analytic methods, numerical solutions of the transverse beam envelope equations, and detailed particle simulations. To facilitate the calculations and simulations, it is assumed that the magnet periods are relatively short and therefore $\dot{x}, \dot{y} \ll \dot{z} \approx u_{0}$, where $u_{0}$ is the (approximately constant) beam velocity component along the $z$ axis. This, in turn, is used to justify use of the paraxial ray approximation $d / d t \leftrightarrow u_{0} d / d z$ and the transformation $t$ $\approx z / u_{0}$ in all analyses. The theoretical analyses of Secs. II, III C, and III D also assume uniform beam density and laminar beam transport (linear space charge forces), while this assumption is naturally relaxed in the numerical particle simulations of Secs. III E and IV. It is further noted that all formulas and calculations assume nonrelativistic beams. Generalization to relativistic expressions is illustrated for infinitely wide sheet beams in Ref. 20.

This work complements prior work ${ }^{19}$ on PCM focusing of semi-infinite sheet beams (i.e., focusing in the narrow transverse dimension only) by comparing the envelope stability of the PCM-focused sheet beam with previous analyses of PCM-focused round beams. More importantly, this work addresses the issue of beam edge confinement for finitewidth sheet beams. For the latter issue, two complementary magnet configurations are investigated: (1) the offset-pole PCM configuration, ${ }^{18,20}$ and (2) a hybrid periodic scheme combining a planar PCM array for bulk beam confinement with periodic-quadrupole-magnetic (PQM) fields for beam edge focusing. With either approach, robust side focusing is observed for remarkably high-space-charge beams. It is also

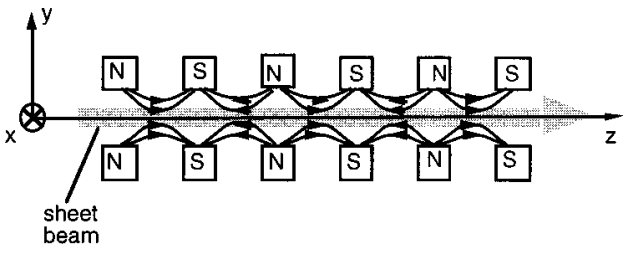

FIG. 1. Simplified illustration of semi-infinite (uniform and infinite along $x$ ), planar, periodically cusped magnetic (PCM) field configuration for focusing of sheet electron beams in their narrow transverse dimension.

shown with analytical expressions and numerical analyses that the magnetic fields required in these configurations for sheet beam focusing are within the capabilities of modern permanent magnet technology.

\section{PCM-FOCUSED SEMI-INFINITE SHEET BEAM ENVELOPE STABILITY}

Consider a semi-infinite PCM configuration (uniform and infinite along $x$ ) similar to that shown in Fig. 1. In the magnet gap, the magnetic fields can be approximately represented by ${ }^{18}$

$$
\begin{aligned}
& B_{y, m}(y, z) \approx-B_{0} \sinh \left(k_{m} y\right) \cos \left(k_{m} z\right), \\
& B_{z, m}(y, z) \approx+B_{0} \cosh \left(k_{m} y\right) \sin \left(k_{m} z\right),
\end{aligned}
$$

where $B_{0}$ is the on-axis magnitude of the field, $k_{m}$ $=2 \pi / \lambda_{m}$, and $\lambda_{m}$ is the spatial period of the magnet array. For a thin beam with half thickness $y_{b} \ll y_{m}$ (where $y_{m}$ is the half height of the magnet gap), one can simplify

$$
\begin{aligned}
& B_{y, m}(y, z) \approx-B_{0} k_{m} y \cos \left(k_{m} z\right), \\
& B_{z, m}(z) \approx+B_{0} \sin \left(k_{m} z\right) .
\end{aligned}
$$

The time-dependent equations of motion for an electron of charge $-e$, mass $m$, under the influence of the above magnetic fields and a self-space-charge electric field $E_{y, s}$ can be written as

$$
\begin{aligned}
& \ddot{x}=-\omega_{c z}(z) \dot{y}+\omega_{c y}(y, z) u_{0}, \\
& \ddot{y}=-\frac{e}{m} E_{y, s}+\omega_{c z}(z) \dot{x},
\end{aligned}
$$

where $\omega_{c y}=e B_{y, m}(y, z) / m, \omega_{c z}=e B_{z, m}(z) / m$, and for a semi-infinite sheet beam the self-electric field in the $x$ direction is taken as approximately zero. To further facilitate the analysis, we consider a uniform beam density in $y$ and laminar beam transport for which

$$
E_{y, s} \approx-(m / e) \omega_{p 0}^{2} y_{b 0},
$$

where $\omega_{p 0}^{2} \equiv e^{2} n(0) / m \epsilon_{0}$ is the usual beam plasma frequency, $\epsilon_{0}$ is the permittivity of free space, and $n(0)$ and $y_{b 0}$ are the beam density and beam half thickness, respectively, at $z=0$.

The coupled equations of motion (5) and (6) were numerically solved within the paraxial ray approximation and the assumptions of Eq. (7). A plot of the resulting electron motion is shown in Fig. 2, and illustrates several expected features of electron motion in periodic fields. This simulation is for a $10 \mathrm{kV}$ beam with an initial current density of 12 

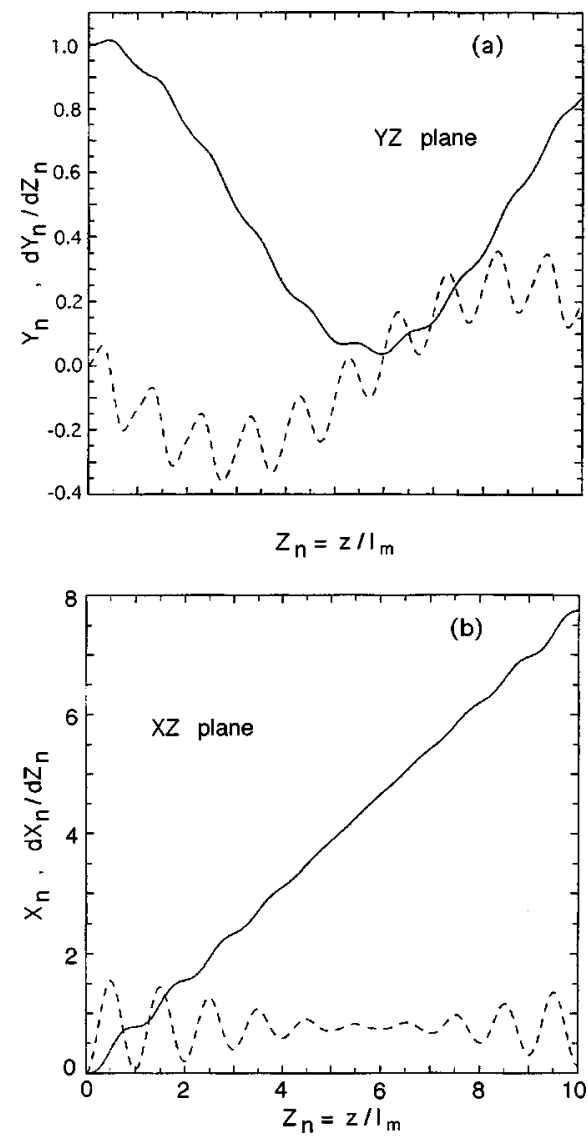

FIG. 2. Plots of the normalized electron position (solid curves) and velocities (dashed curves) vs axial position (normalized to the magnet period) in the semi-infinite PCM array. (a) Normalized position and velocity in the narrow (or $y$ ) transverse dimension. The position is normalized with respect to initial minor radius, $y_{b}$, and the velocity is normalized with respect to injection velocity, initial minor radius, and magnet period: $d Y_{n} / d Z_{n}$ $=(d y / d t) \lambda_{m} / u_{0} y_{b}$. (b) Normalized position and velocity in the wide (or $x$ ) transverse dimension. The position is normalized with respect to initial major radius, $x_{b}$, and the velocity is normalized with respect to injection velocity, initial minor radius, and magnet period: $d X_{n} / d Z_{n}$ $=(d x / d t) \lambda_{m} / u_{0} x_{b}$. The high-frequency oscillations correspond to the periodicity of the magnets, while the low-frequency oscillations correspond to betatron oscillations for the unmatched beam.

$\mathrm{A} / \mathrm{cm}^{2}$, focused by a PCM field having a $3.75 \mathrm{~mm}$ period and a field amplitude of $700 \mathrm{G}$. In Fig. 2, the $z$ axis has been normalized to the magnet period $\left(Z_{n}=z / \lambda_{m}\right)$ and the transverse coordinates have been normalized to the initial beam half-thickness $\left(Y_{n}=y / y_{b}, X_{n}=x / y_{b}\right)$. The motion in the $X-Z$ plane is a combination of a linear $E \times B$ drift with the highfrequency wiggle motion due to the PCM. Also occuring are slow time-scale oscillations at the betatron frequency $\omega_{\beta}$ $=e B_{0} / \sqrt{2} \mathrm{~m}$. The oscillations are more readily apparent in the $Y-Z$ plane and can be seen to have a period of roughly 12 magnet periods.

Betatron oscillations in the sheet beam lead to a breathing, or ripple effect of the beam thickness, since for a socalled "cold" beam with negligible transverse emittance, the electrons oscillate in phase. It is desirable to minimize this effect through "matching" of the beam betatron oscillations with plasma oscillations due to space-charge. For a semiinfinite sheet beam, the condition for beam matching is given by $\omega_{p 0} \approx \omega_{c 0} / \sqrt{2}=\omega_{\beta}$, where $\omega_{c 0}=e B_{0} / m$. For the beam
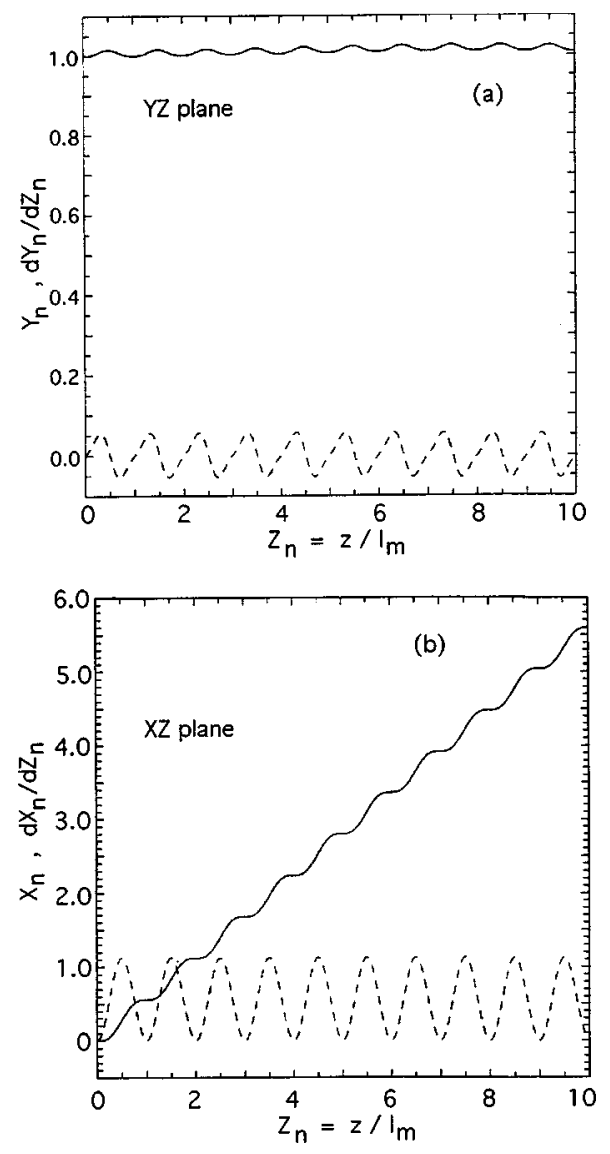

FIG. 3. Matched beam transport for $B_{0}=507 \mathrm{G}$ for the configuration indicated in Fig. 2. Note the absence of betatron oscillations. Again, the solid curves represent normalized position and the dashed curves the normalized velocity. Normalizations are the same as those in Fig. 2.

parameters of Fig. 2, this criteria gives $B_{0}=507 \mathrm{G}$ for a matched condition; Fig. 3 shows the resulting elimination of the betatron motion. The remaining oscillation is solely due to the PCM fields and this represents the minimum ripple condition on the beam.

As the magnet period increases, the beating of the biharmonic motion of the beam comprised of betatron and PCM oscillations can lead to a condition where the PCM oscillation period is comparable to the betatron period, or harmonics of the betatron period. Constructive interference between the two oscillations creates large amplitude, or completely unconfined, orbits which lead to beam loss to the transport channel. This situation is similar to the case for round beams in permanent periodic magnet (PPM) focusing treated by Mendel et al. ${ }^{21}$ In Ref. 21, the radial equation of motion for edge electrons in a round beam was recast in the form of Matthieu's equation. Tabulated solutions for Matthieu's equation showed the existence of a series of passbands and stopbands as the PPM period was increased corresponding to transport of the beam through the focusing stack or loss of the beam to the channel walls. Similarly, for the semi-infinite sheet beam case, the numerical solutions for the trajectory of electrons at the beam edge (envelope calculations) also show the existence of higher order passbands which correlate very well with the Matthieu's solutions. Mathematically, this can be seen if we transform Eqs. (5) and 
(6) into the time domain using $z \rightarrow u_{0} t$, consistent with the paraxial approximation. Further advantage comes from separating time scales under the assumption that betatron motions and space-charge-induced motions evolve on a slow time scale relative to the motion due to the oscillating magnetic field in the short period PCM array. Writing $x=x_{f}+x_{s}$ and $y=y_{f}+y_{s}$ (where $f$ and $s$ subscripts represent fast and slow time-scale motions, respectively), substituting into the equations of motion, separating the fast and slow time scales, and performing various mathematical manipulations yields the approximate result: ${ }^{22}$

$$
\ddot{y}_{s} \approx \frac{e}{m} E_{y, s}+\omega_{c 0}^{2} \cos ^{2}\left(\omega_{m} t\right) y_{s} .
$$

This can be rewritten using the relation $\cos ^{2}(\theta)=1 / 2[1$ $-\cos (2 \theta)]$ :

$$
\ddot{y}_{s} \approx-\frac{e}{m} E_{y, s}+\frac{\omega_{c 0}^{2}}{2}\left[1-\cos \left(2 \omega_{m} t\right)\right] y_{s},
$$

which is of the same form as the Matthieu's equation given in Ref. 21.

The numerically calculated envelope trajectory simulations and calculations based on Eq. (9) (using the tabulated values from Ref. 21) agree quite well on predicting the positions of the pass and stop bands. For the beam parameters used in Fig. 2, the first stopband occurs at $\lambda_{m}=35 \mathrm{~mm}$ and the second passband occurs between 60 and $80 \mathrm{~mm}$. The relatively poor focusing available in the higher passbands, leading to larger amplitude electron orbits, probably overcomes any technological advantage to be gained by using the easier-to-fabricate longer magnet periods.

It should also be noted that, besides the similarities of the pass and stop bands for beam envelope stability, the similarities between planar PCM and round-beam-PPM focusing systems carries over into the basic scalings required to achieve beam focusing. In general, the magnet period $\lambda_{m}$ must be smaller than the scale length associated with plasma oscillations, $\lambda_{p}=2 \pi u_{0} / \omega_{p}$, in order to focus the beam against space charge. Typically, in PPM systems, a ratio of $\lambda_{m} / \lambda_{p}<0.33$ is desirable ${ }^{23}$ and our envelope simulations indicate that a comparable scaling is required in planar PCM focusing of sheet beams. In addition, the magnet period should be smaller than the betatron scale length, $\lambda_{\beta}$ $=2 \pi u_{0} / \omega_{\beta}$, in order to avoid large amplitude oscillation and beam current loss. Consideration of the point at which the first stopband occurs for planar-PCM-focused sheet beams gives the scaling $\lambda_{m} / \lambda_{\beta}<0.25$, essentially the same as that for PPM-focused round beams. ${ }^{21}$

\section{OFFSET-POLE PCM FOR BEAM EDGE FOCUSING}

\section{A. Analytic magnetic field expressions}

Consider the offset-pole configuration previously proposed in Refs. 18, 20 and illustrated in Fig. 4. Near the center of the array the magnetic fields can be approximately represented by Eqs. (1) and (2) and these field components provide diocotron-instability-free focusing of semi-infinite sheet-electron beams for short PCM periods, as demonstrated in Ref. 19. Side focusing in the larger transverse di-
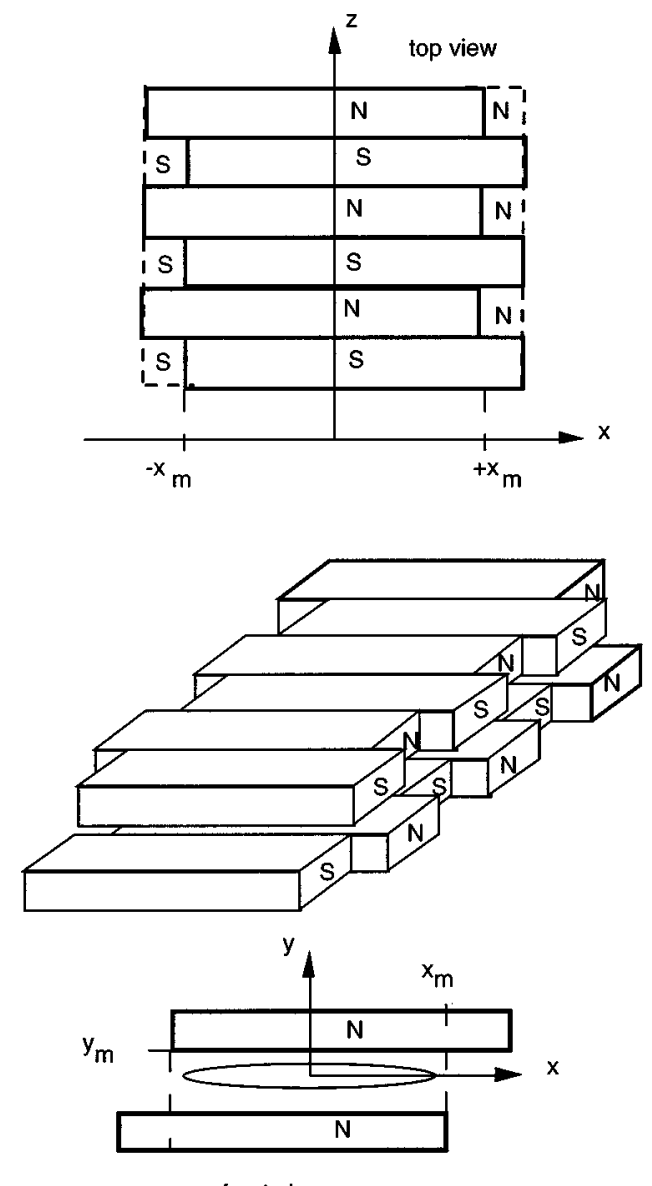

front view

FIG. 4. Schematic of the basic configuration of the offset-pole PCM array. In contrast to planar wigglers used for free electron laser experiments, the PCM has the same magnet polarity across the midplane. An offset in the magnets produces side focusing in the wide dimension of the beam.

mension of the beam in this configuration should be provided by the fringe fields of this array. ${ }^{18}$ Some support for this hypothesis can be based on experimental studies of offsetpole planar wiggler focusing of high-voltage sheet beams with very low space charge,${ }^{20,24}$ but investigations of offsetpole planar PCM confinement of low-voltage, high-spacecharge beams have never been reported. Analytic expressions for the fringe fields of offset-pole configurations can be obtained by replacing the offset-pole regions with semiinfinite sheets of "surface magnetic charge", as discussed in Ref. 18. The side fields are thus calculated as integrals over the surface charge:

$$
\begin{aligned}
& B_{x, s}(x, y)=C_{b} \int \rho_{m} \frac{\left(x-x^{\prime}\right)}{\left[\left(x-x^{\prime}\right)^{2}+\left(y-y^{\prime}\right)^{2}\right]} d x^{\prime}, \\
& B_{y, s}(x, y)=C_{b} \int \rho_{m} \frac{\left(y-y^{\prime}\right)}{\left[\left(x-x^{\prime}\right)^{2}+\left(y-y^{\prime}\right)^{2}\right]} d y^{\prime},
\end{aligned}
$$

where $C_{b}$ is a dimensioning constant. The equivalent magnetic surface charge density, $\rho_{m}$, can be written as

$\rho_{m}\left(x^{\prime}, y^{\prime}\right)= \begin{cases}-\rho_{0}\left[\delta\left(y^{\prime}-y_{m}\right)-\delta\left(y^{\prime}+y_{m}\right)\right] & \text { for } x>0, \\ +\rho_{0}\left[\delta\left(y^{\prime}-y_{m}\right)-\delta\left(y^{\prime}+y_{m}\right)\right] & \text { for } x<0 .\end{cases}$ 


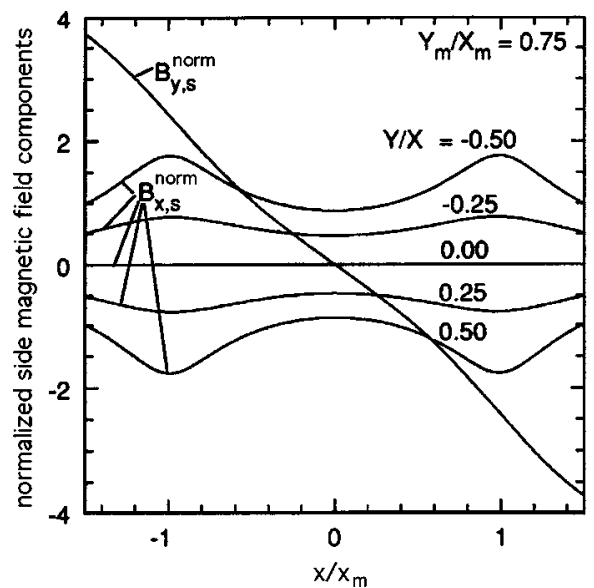

FIG. 5. Plot of the normalized side fields $B_{x, s}^{\text {norm }} \equiv B_{x, s} \pi / B_{s, 0}$ and $B_{y, s}^{\text {norm }}$ $\equiv B_{y, s} \pi / B_{s, 0}$ for several aspect ratios $y_{m} / x_{m}$. The horizontal axis is the $x$ coordinate normalized to the magnet width, $x_{m}$.

Substituting Eq. (12) into Eqs. (10) and (11) and integrating over all magnetic charge sheets gives

$$
\begin{aligned}
B_{x, s}= & \frac{B_{s 0}}{4 \pi}\left\{\ln \left[\left(x-x_{m}\right)^{2}+\left(y+y_{m}\right)^{2}\right]-\ln \left[\left(x-x_{m}\right)^{2}\right.\right. \\
& \left.+\left(y-y_{m}\right)^{2}\right]-\ln \left[\left(x+x_{m}\right)^{2}+\left(y-y_{m}\right)^{2}\right] \\
& \left.+\ln \left[\left(x+x_{m}\right)^{2}+\left(y+y_{m}\right)^{2}\right]\right\}, \\
B_{y, s}= & \frac{B_{s 0}}{2 \pi}\left[\tan ^{-1}\left(\frac{x-x_{m}}{y-y_{m}}\right)-\tan ^{-1}\left(\frac{x-x_{m}}{y+y_{m}}\right)\right. \\
& \left.+\tan ^{-1}\left(\frac{x+x_{m}}{y-y_{m}}\right)-\tan ^{-1}\left(\frac{x+x_{m}}{y+y_{m}}\right)\right],
\end{aligned}
$$

where $B_{s 0}=C_{b} \rho_{0}$ is the limiting magnitude of $B_{y, s}$ for $x$ $\gg x_{m}$. Plots of Eqs. (13) and (14) are shown in Fig. 5 for several magnet array aspect ratios $\left(y_{m} / x_{m}\right)$. These plots help in understanding the dynamics of side focusing in the offsetpole PCM configuration. Focusing of the beam in the wide transverse dimension (in $x$ ) is provided by the $u_{0} B_{y, s}$ force, where $u_{0}$ is the beam drift velocity along $z$. The linear region of $B_{y, s}$ near the $x=0$ midplane provides a force balance between magnetic focusing and the approximately linear space-charge electric field and, as discussed below, theoretically provides for beam matching in the wide transverse direction. The $B_{x, s}$ component is zero along the midplane but off the midplane the polarity of $B_{x, s}$ is such that particles are pushed away (defocused) along the $y$ direction. However, these adverse effects of the $B_{x, s}$ components can be overcome for very thin beams $\left(y_{b} \ll x_{b}<x_{m}\right)$ where particles are close to the axis all along the midplane, and especially for highly elliptic sheet beams where the tapering of the beam at the edge reduces defocusing.

\section{B. Three-dimensional magnet simulations of the offset-pole PCM}

The three dimensional (3D) finite element magnetostatic code TOSCA $^{25}$ was used to model the offset-pole PCM and check the analytic expressions for the side fields. The available version of TOSCA could not model the permanent mag-

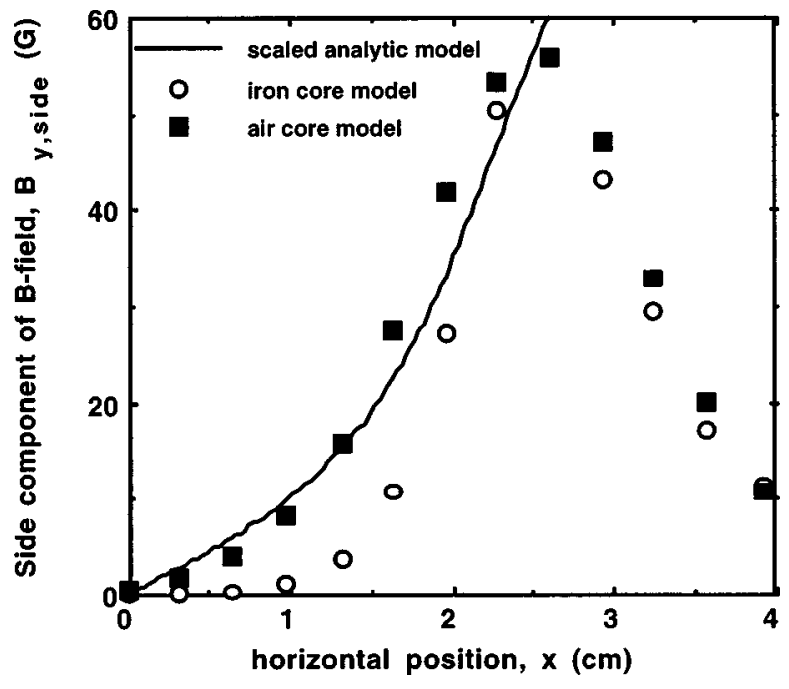

FIG. 6. Predicted $B_{y, s}$ side fields for the offset-pole PCM array as a function of the transverse dimension, $x$. The iron-core array model corresponds to an illustrative coil excitation ( $\mathrm{mmf}$ ) of $1000 \mathrm{amp}$-turns. The air-core coil model results and the analytic model were scaled to yield the same field at $x$ $=2.6 \mathrm{~cm}$ as the iron-core coil model. Good agreement with the analytic model for permanent magnets is obtained with the simulated air-core numerical computations, justifying the use of the analytic model in subsequent particle simulations.

net PCM assumed in the analytic calculation of the side fields leading to Eqs. (13) and (14). Instead, air-core electromagnet models were used as an approximation to a magnet array constructed from high-remanance permanent magnet materials and compared with the predicted fields for an ironcore magnet array. In the latter case, the $B-H$ curve for iron polepieces was taken from the 1010 steel table used in the POISSON/PANDIRA ${ }^{26}$ set of two-dimensional (2D) magnet design codes. For illustration, the array width and height and the magnet period were chosen to be $x_{m}=2.0 \mathrm{~cm}, y_{m}$ $=0.75 \mathrm{~cm}$, and $\lambda_{m}=3.0 \mathrm{~cm}$, respectively. Individual magnet cores were selected to have dimensions $(w \times h \times t)$ of 5.0 $\times 1.75 \times 0.5 \mathrm{~cm}$. The inner spacing between cores along $z$ was taken as $2.0 \mathrm{~cm}$. Each magnet coil was offset $0.75 \mathrm{~cm}$ from the array midplane with rectangular cross-section dimensions $(w \times h)$ of $0.75 \times 1.25 \mathrm{~cm}$. The inner transverse dimensions of the coil were chosen to be the same as the width and thickness of the cores.

The calculated $B_{y}$ side fields from these simulations, sampled along the $y=0$ midplane at a fixed $z$ position, are plotted in Fig. 6, along with a scaled version of the analytic result of Eq. (14). The critical issue to compare is the degree to which the numerically simulated arrays produce a $B_{y \text {,side }}$ field component that varies approximately linearly with $x$, as needed for force balancing in this dimension, and consistent with the expectation derived from the analytic formula, Eq. (14). Hence, having calculated the $B_{y \text {,side }}$ field for the ironcore array with an illustrative choice of coil $\mathrm{mmf}$ of 1000 amp-turns, the air-core simulation's amp-turns were adjusted until it produced the same peak field at $x=2.6 \mathrm{~cm}$. Similarly, the analytic model of Eq. (14) was scaled until also producing the same value at $x=2.6 \mathrm{~cm}$. Fairly good agreement is observed between the analytic model and the air-core model of the permanent magnet array, especially, for the central 
region where $x<1.4 \mathrm{~cm}$. However, it is apparent that the iron-core electromagnet suffers from a fairly weak field (and weak focusing force) near $x=0$, and has an abrupt, nonlinear increase starting at about $x=1.0 \mathrm{~cm}$. As a result, the agreement between the iron-core array model and the analytic model of Eq. (14) is relatively poor, especially in the central region near $x=0$. The reason for these observations is that within the air-core coil the flux is more evenly distributed (for a long, thin coil) than is the case for iron. Hence, the air-core case more closely resembles the permanent magnet configuration and the assumptions used in the derivation of Eq. (14) are better modeled with an air-core magnet. This justifies the use of the analytic model in subsequent particle simulations, described below.

Since magnet tunability may be desirable in some cases (e.g., laboratory experiments), and the presence of a high- $\mu$ material such as iron greatly enhances the achieved field for a given applied current, it still may be beneficial to use ironcore arrays. However, it should be noted that, recalling the discussion in the previous section, beam matching will be more difficult to achieve without a linear $B_{y, s}$ field region to balance linear space-charge forces along the $x$ axis of the beam and magnet array.

\section{Sheet beam matching with offset-pole PCM confinement}

As noted, the $B_{y, s}$ side field of Eq. (14) is approximately linear around the point $x=0$. For points near the midplane $(y=0)$, assuming a very wide magnet array with $y_{m} / x_{m}$ $\ll 1$, and using the expansion $\tan ^{-1}(\Psi+\epsilon) \approx \pi / 2-1 / \Psi(1$ $-\epsilon / \Psi)$, one can obtain

$$
B_{y, s}(x, 0) \approx-\frac{2 B_{s 0}}{\pi}\left(\frac{y_{m}}{x_{m}^{2}}\right) x .
$$

If we further assume highly elliptic sheet-beam cross sections both for their analytically tractable self-fields, ${ }^{18}$ as well as their practical advantage of reduced beam density and defocusing forces at the beam edges, we can arrive at a force-balance condition for edge confinement: ${ }^{22}$

$$
B_{s 0} \approx \frac{\pi \omega_{p 0}^{2}}{2 u_{0}}\left(\frac{y_{b} / x_{b}}{y_{m} / x_{m}}\right) x_{m} .
$$

From the above equation it is evident that the required side magnetic field amplitude can be held to reasonably small values provided that the beam aspect ratio $y_{b} / x_{b}$ is made small. Fortunately, this condition is the same as required to avoid defocusing effects from the $B_{x, s}$ magnetic field term, implying that high current densities can be transported in highly elliptic beams using moderate side field magnitudes.

\section{Sheet beam tilt in offset-pole PCM focusing}

One other adverse effect of the addition of (effectively) uniform (nonperiodic) side-focusing fields to the periodic center PCM fields is a resultant tilt in the beam midplane off of the magnet array midplane, as illustrated in Fig. 7. This can be understood by noting that the addition of uniform side field components to the periodic center fields causes a rotation in the axis along which the total $B_{y}$ component of the

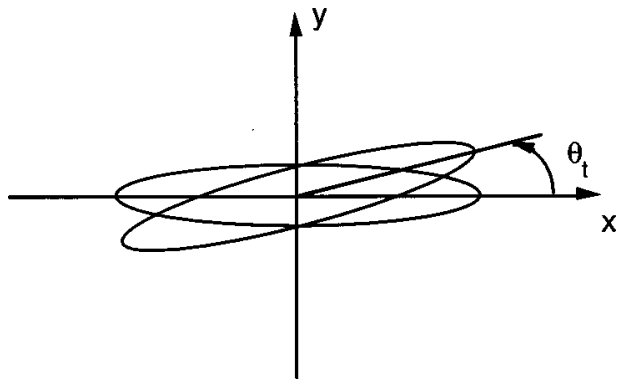

FIG. 7. Cross-sectional illustration of beam tilt that can occur in offset-pole PCM focusing.

magnetic field is zero. In other words, since the axially uniform side field component changes polarity across the $x=0$ plane, yet the center PCM fields are symmetric, the beam will settle into an equilibrium around the null field axis. Using the linear approximation to the side $B_{y, s}$ field in Eq. (15) and noting that the period average of Eq. (1) is $k_{m} y B_{0} / \sqrt{2}$ near the midplane, we have the following expression for lines of constant magnetic field:

$$
\left.B_{y}(x, y \approx 0)\right|_{\text {constan }} \approx \frac{1}{\sqrt{2}} B_{0} k_{m} y-B_{s 0} \frac{2 y_{m}}{\pi x_{m}^{2}} x .
$$

The null field axis occurs at an angle given by

$$
\theta_{0}=\tan ^{-1}(y / x)=\tan ^{-1}\left(\frac{2 \sqrt{2}}{\pi k_{m} x_{m}} \frac{y_{m}}{x_{m}} \frac{B_{s 0}}{B_{0}}\right) \text {. }
$$

For most beams of interest, the small amount of tilt incurred is probably not significant. For example, anticipating beam simulations in following sections, if we have $y_{m} / x_{m}$ $=1 / 16, \lambda_{m} / x_{m}=0.3 / 4$, and $B_{s 0} / B_{0}=1 / 2$, then Eq. (18) gives $\theta_{0} \approx 0.33 \mathrm{mrad}$. A highly elliptic sheet electron beam with an aspect ratio of $x_{b} / y_{b}=56$ would thereby have its effective thickness increased by $\theta_{0} x_{b} / y_{b}$, or about $2 \%$. Only in cases of highly elliptic beams having extreme space charge, requiring large side magnetic fields for focusing, would beam tilt be expected to be problematic in pushing the beam edges far enough off-axis into the defocusing $B_{x, s}$ field.

\section{E. Particle simulations with offset-pole PCM edge confinement}

The 2-1/2-D particle-in-cell (PIC) code MAGIC ${ }^{27}$ was used to study sheet-beam focusing in offset-pole PCM fields. As before, the $z$ drift coordinate was transformed to the time domain using $z \rightarrow u_{0} t$ according to the paraxial approximation. The uniform (static) side magnetic fields given in Eqs. (13) and (14) were superimposed upon the central PCM fields (for thin sheet beams) of Eqs. (3) and (4).

For illustration purposes, the beam considered here is a highly elliptical sheet beam with major and minor radii of $x_{b}=1.4 \mathrm{~cm}$ and $y_{b}=0.025 \mathrm{~cm}$, respectively (note $x_{b} / y_{b}$ $=56$ ), with a total beam current of $I_{b}=10 \mathrm{~A}$ and a beam energy of $e V_{b}=10 \mathrm{keV}$. The current, space charge, and particle densities of the beam correspond to $90.9 \mathrm{~A} / \mathrm{cm}^{2}, 15.5$ $\mathrm{mC} / \mathrm{m}^{3}$, and $9.7 \times 10^{16} \mathrm{~m}^{-3}$, respectively. For the simulations the beam is initially loaded with a Gaussian velocity distribution in all three velocity coordinates. The rms axial veloc- 


\section{Offset-Pole PCM}

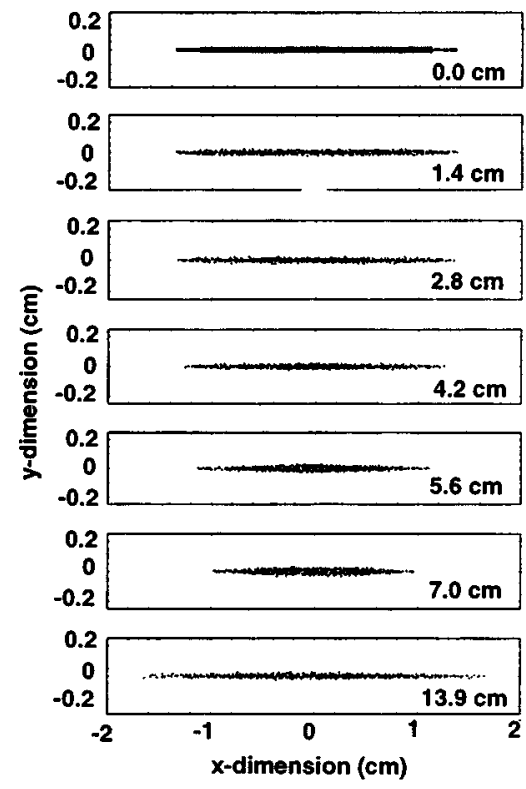

(a)

\section{Conventional Solenoid}

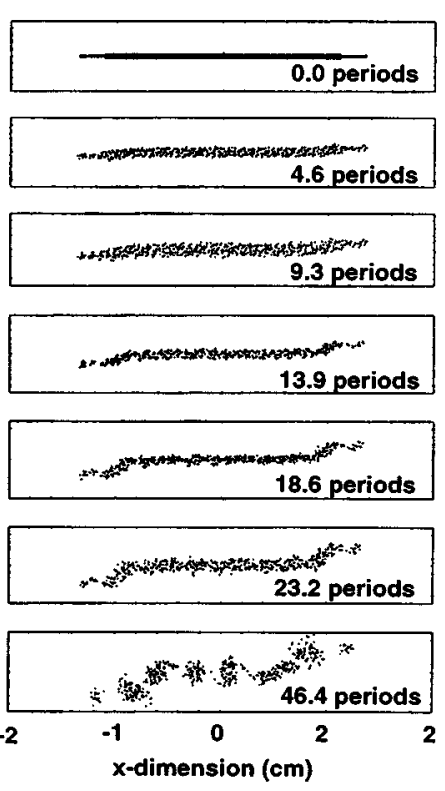

(b)

\section{PCM-PQM Hybrid}

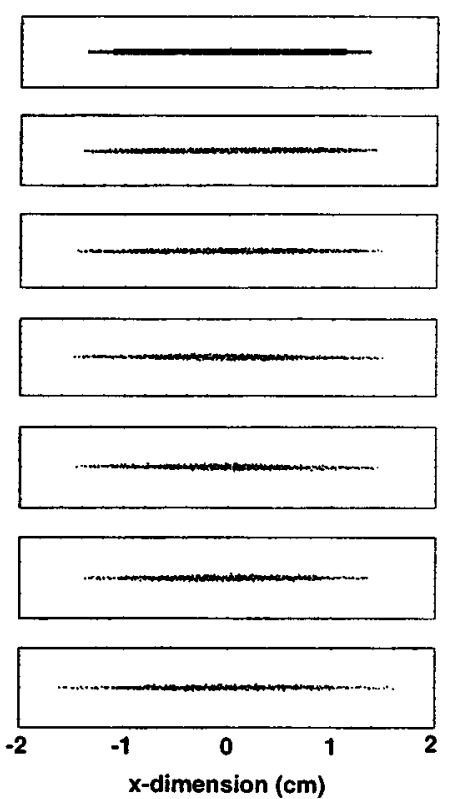

(c)

FIG. 8. Cross-sectional particle plots from PIC simulations of a high-space-charge density elliptical sheet electron beam (with $x_{b}=1.4 \mathrm{~cm}, y_{b}=0.025 \mathrm{~cm}$, $I_{b}=10 \mathrm{~A}, V_{b}=10 \mathrm{kV}$ ) in various magnetic field focusing configurations. (a) An offset-pole PCM array. The magnet array has $\lambda_{m}=3 \mathrm{~mm}, B_{0}=1200 \mathrm{G}$, $B_{s 0}=534 \mathrm{G}, x_{m}=4.0 \mathrm{~cm}$, and $y_{m}=0.5 \mathrm{~cm}$. (b) The same beam in a uniform magnetic field with $B_{0}=1200 \mathrm{G}$. (c) The same beam in a hybrid PCM-PQM array. The magnet array has $\lambda_{m}=3 \mathrm{~mm}, B_{0}=1200 \mathrm{G}, B_{q} / x_{m}=3500 \mathrm{G} / \mathrm{cm}, x_{m}=4.0 \mathrm{~cm}$, and $y_{m}=0.5 \mathrm{~cm}$.

ity spread (ratio of $v_{\text {th }, z} / u_{0}$ where $v_{\text {th }, z}$ is the thermal velocity along $z$ ) is taken to be $1 \%$, which is consistent with, but slightly larger than, the velocity spread typically produced in Pierce-type linear beam sources. Equipartition of the velocity distribution is assumed, i.e., the rms thermal spread in the transverse components is also taken to be $v_{\text {th }, x} / u_{0}$ $=v_{\text {th }, y} / u_{0}=1 \%$. Conducting wall boundary conditions are invoked at $x= \pm 2 \mathrm{~cm}$ and $y= \pm 0.25 \mathrm{~cm}$ (laboratory frame, consistent with the nonrelativistic analysis).

Figure 8(a) illustrates the evolution of the beam cross section in offset-pole PCM focusing, i.e., the magnetic focusing fields of Eqs. (3), (4), (13), and (14). Magnetic field parameter value choices include $\lambda_{m}=3 \mathrm{~mm}, B_{0}=1200 \mathrm{G}$, $B_{s 0}=534 \mathrm{G}, x_{m}=4.0 \mathrm{~cm}$, and $y_{m}=0.5 \mathrm{~cm}$. The beam crosssection images are shown for simulation times corresponding to (top-to-bottom) $\tau=0.0,0.24,0.48,0.72,0.95,1.2$, and 2.4 ns. Using the $z \leftrightarrow t$ transformation discussed previously, these times correspond to axial locations $z=0.0,1.4,2.8$, $4.2,5.6,7.0$, and $13.9 \mathrm{~cm}$, or normalized axial locations $Z_{n}$ $=z / \lambda_{m}=0.0,4.6,9.3,13.9,18.6,23.2$, and 46.4 magnet periods, respectively [as indicated in the inset frame labels in Figs. 8(a) and 8(b)].

The particle plots of Fig. 8(a) demonstrate several points. First, relatively high-space-charge beams can be focused without instability with modest, and readily achievable, magnetic field magnitudes. Even more signficant is the total, stably confined beam current realized for a given beam voltage. Here we note that the total beam perveance is a considerable $I_{b} / V_{b}^{3 / 2}=10 \mu$ pervs. For comparison, the PPMfocused $50 \mathrm{MW}$ klystron round beam of Ref. 28 has a total beam perveance of $350 \mathrm{~A} /(440 \mathrm{kV})^{3 / 2}=1.2 \mu$ pervs. Since the perveance of the sheet beam configuration is obtained for low beam voltages, the possibility of realizing high-power, compact systems through high total current rather than with high voltage (and bulky high voltage power supplies) becomes evident. Another point illustrated by the simulations is the robust stability of the sheet-beam confinement in offsetpole PCM arrays, indicated by the long-term confinement (>40 magnet periods). Finally, beam matching appears to be challenging to simultaneously achieve in both transverse dimensions. For example, the beam of Fig. 8(a) is relatively well matched in the thin transverse dimension. However, in the other (wide) transverse plane the beam oscillates or breathes on a time scale that is slow compared to the PCM field periodicity, $\omega_{m}^{-1}=\left(k_{m} u_{0}\right)^{-1}$, due to a mismatch of focusing and space-charge forces in this plane. Achieving an acceptable force balance or beam matching in the wide transverse plane represents a remaining research challenge for offset-pole PCM focusing of high-space-charge sheet beams.

For comparison purposes, Fig. 8(b) shows the same beam in a static, uniform solenoidal magnetic field oriented along the $z$ axis (pointing out of the page). The field magnitude, $B_{z 0}$ is taken to equal the peak PCM magnetic field used in Fig. 8, or $B_{z 0}=1200 \mathrm{G}$. Recalling that the growth rate of the diocotron instability scales as $\omega_{p}^{2} / 2 \omega_{c}$, or as the ratio of the charge density, $n e$, to the magnetic field amplitude $B_{z 0}$, we note that by using the peak PCM field rather than the rms value of the sinusoidal PCM field (i.e., $B_{0} / \sqrt{2}$ ) we are, in effect, taking the "best case" condition of solenoidal focus- 
ing for this comparison. Still, Fig. 8(b) shows that the beam begins to shear and tilt early on in the simulation and eventually filaments and scrapes the transport channel walls near the end of the simulation.

To further confirm the capability of the offset-pole PCM array for focusing very high-perveance sheet beams, a case was studied wherein the beam current was scaled by a factor of 5 (to $50 \mathrm{~A}$ ) for the same size beam as in Figs. 8(a) and 8 (b). This corresponds to a beam with current density, space charge, and particle densities of $455 \mathrm{~A} / \mathrm{cm}^{2}, 77.5 \mathrm{mC} / \mathrm{m}^{3}$, and $4.9 \times 10^{17} \mathrm{~m}^{-3}$, respectively. Based on the scalings in Eqs. (6), (7), and (16), confinement of such a beam should require commensurately scaled increases in the peak magnetic field parameters $B_{0}$ and $B_{s 0}$. This was indeed found to be the case, and stably confined sheet beams were observed ${ }^{22}$ for choices of $B_{0}=4000 \mathrm{G}$ (considered to be the upper achievable limit of current permanent magnet technology) and $B_{s 0}=2515 \mathrm{G}$. The results look very similar to the lowerdensity, lower-field case of Fig. 8 (consistent with the scaling arguments), and are not displayed here. Again, however, stable focusing is realized in both transverse planes leaving one with the rhetorical question of just how such a ultrahighspace-charge beam might be generated.

While the above cases illustrate the physical capabilities of offset-pole PCM focusing, the choice of the array period $\left(\lambda_{m}=3 \mathrm{~mm}\right)$ may pose a fabrication challenge. To investigate alternative choices, a simulation was conducted of the beam of Fig. 8(a) propagating in a offset-pole PCM magnet array having $\lambda_{m}=1 \mathrm{~cm}$, but retaining otherwise identical parameter choices (i.e., $B_{0}=1200 \mathrm{G}, B_{s 0}=534 \mathrm{G}$, etc.). In spite of the large magnet period, the beam was observed to remain robustly stable. ${ }^{22}$ The only perceptible difference was an enhanced tilt in the beam midplane which, recalling the discussion of beam tilt and the scaling of $\theta_{0} \sim \lambda_{m}$, is to be expected if only the magnet period is increased. Even in this simulation case, however, the tilt angle was less than a few degrees, which could have been considerably reduced either by increasing $B_{0}$ or by widening the magnet array.

\section{HYBRID PCM-PQM SHEET BEAM FOCUSING}

A more flexible focusing scheme for sheet electron beams is a hybrid structure consisting of a PCM array in the center and canted (quadrupolar), periodically varying side arrays for beam edge focusing, hereafter referred to as PCMPQM focusing. This hybrid structure is illustrated in Fig. 9. The basic mechanism for edge focusing is similar to the focusing mechanism in periodic quadrupole magnet arrays, as discussed in Ref. 29, where a gradient in the side fields in required to focus against beam space charge.

The magnitude of the focusing force from canted periodic quadrupole magnets at the beam edge may be written as $F_{x} \sim e u_{0} B_{y}^{\prime} x_{b}$ where $B_{y}^{\prime}=\partial B_{y} / \partial x$ is the gradient in the $y$ component of the field under the canted poles at the edge. Since this edge focusing makes use of the dominant axial velocity component $u_{0}$ and strong field gradients of $4 \mathrm{kG} / \mathrm{cm}$ are readily achievable with $\mathrm{Sm}_{x} \mathrm{Co}_{y}$ or $\mathrm{NdFeBo}$ permanent magnet materials, ${ }^{29}$ this type of edge focusing should be feasible even with very high-space-charge sheet beams.
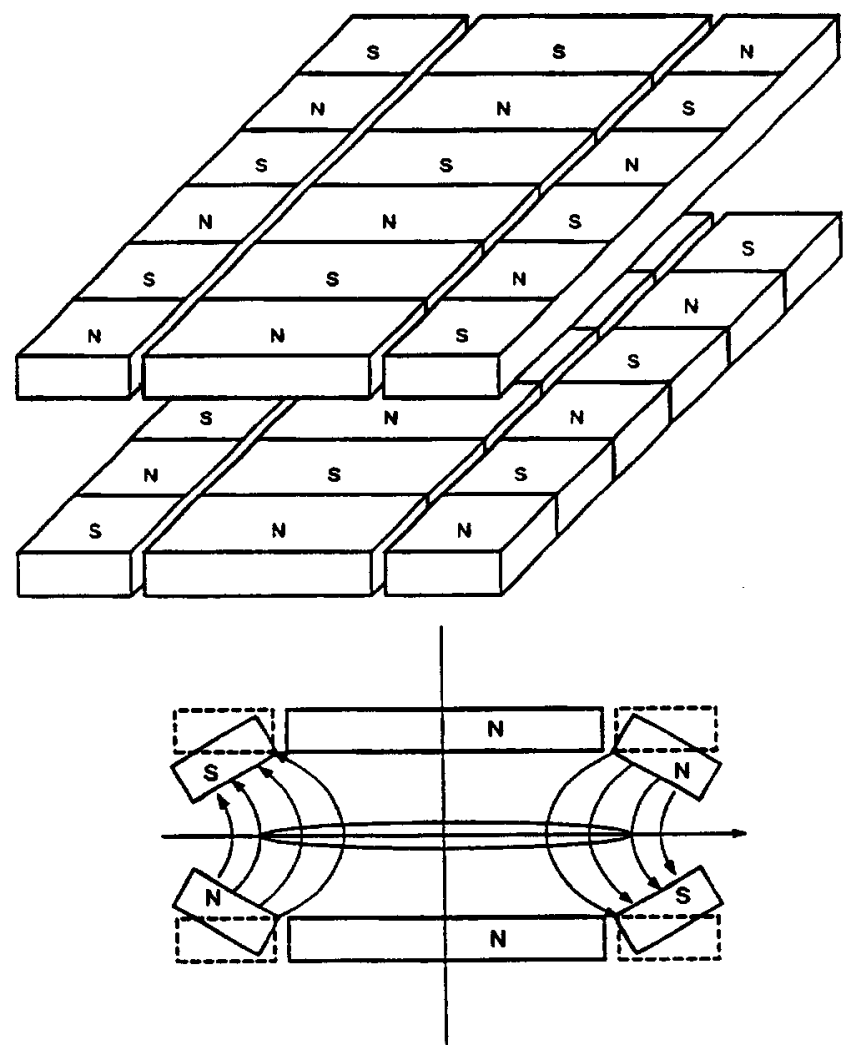

FIG. 9. Quadrupolar side magnetic fields for beam edge focusing are created by tilting side magnet arrays as indicated.

The main advantages of this configuration over the offset-pole configuration of Fig. 4 are twofold. First, beam matching is more easily achieved in both transverse planes since adjustment of the side field gradient can be made through the side magnet cant-angle or spacing independently of the central PCM array. The second advantage is the elimination of the beam tilt problem, which is especially important for relatively "thick" beams where the combination of beam tilt and uniform $B_{x, s}$ defocusing fields (in the offsetpole PCM configuration) can lead to loss of the beam. In contrast, in the hybrid array the side fields are also periodically-varying so the period-averaged magnetic axis remains along the array midplane.

Assuming the usual linear superposition property of permanent magnets we can simply write down the side magnetic fields of the PCM-PQM hybrid as

$$
\begin{aligned}
& B_{x, s}(y, z)=B_{q}\left(\frac{y}{x_{m}}\right) \sin \left(k_{m} z+\phi_{s}\right), \\
& B_{y, s}(x, z)=B_{q}\left(\frac{x}{y_{m}}\right) \sin \left(k_{m} z+\phi_{s}\right),
\end{aligned}
$$

where, again, $x_{m}$ and $y_{m}$ are the PCM magnet array width and height and $k_{m}$ has the same meaning as before. The quadrupole field at the center of the canted poles' surface is $B_{q}$ and $\phi_{s}$ is the phase difference between the PCM and quadrupole stacks.

The MAGIC PIC code was again used to study the focusing in the hybrid array using a superposition of the fields of Eqs. (3), (4), (19), and (20) and the paraxial transforma- 
tion $k_{m} z \rightarrow \omega_{m} t / u_{0}$. In addition, a two-period magnetic field entrance taper was applied to the side fields to reduce the initial tilt experienced by the laminar input beam at the entrance to the array. The taper is implemented by increasing the side-field amplitude by $B_{q} / 4$ over each half period until the full magnitude is reached at the beginning of the third magnet period. Such field tapering is commonly done at the entrance of free electron laser wiggler magnets for similar reasons.

Figure 8(c) illustrates the effectiveness of the PCMPQM hybrid for stable, long-term focusing of high-spacecharge sheet electron beams. Moreover, the beam is more readily matched in the wide transverse dimension and the amplitude of radial oscillations is significantly reduced [i.e., compare with Fig. 8(a)]. This improved facility of matching is a consequence of the linearity of, and independent control one has over the quadrupolar side fields. Another important point is that the beam tilt with respect to the midplane has been eliminated. Similar characteristics of effective PCMPQM sheet-beam confinement can be observed in simulations with longer (and thus more practical) magnet periods (e.g., $\sim 1 \mathrm{~cm})$.

\section{SUMMARY AND CONCLUSIONS}

Theoretical analyses and numerical simulations have been described which further develop the knowledge base regarding periodically focused sheet electron beams. The particular emphasis of this paper was the description of magnetic fields and electron dynamics associated with two-plane focusing of sheet beams in two versions of PCM arrays. One configuration accomplishes side focusing using a periodic offset of the magnet poles in the wide transverse dimension of the beam. The other configuration uses a split PQM array on the two sides to accomplish side focusing.

The analyses demonstrate several points. First, it is shown that the beam envelope stability dynamics in the narrow transverse dimension are fundamentally the same as that for conventional PPM focusing of round-cross-section electron beams, exhibiting precisely the same beam envelope stability and instability bands associated with the coupling between PCM and betatron oscillations. Second, it is shown that both PCM configurations can stably confine sheet electron beams with relatively large space charge (electron particle densities). The analyses indicate that two-plane matched beam equilibria in the offset-pole configuration may be harder to realize, in practice, however, due to the lack of completely independent control over the narrow and wide transverse dimension focusing fields. The challenge of finding these matched beam equilibria is expected to be further exacerbated if iron pole pieces are utilized to increase magnetic flux density, due to the added difficulty this would mean for realizing uniform spatial gradient in the focusing force needed for balancing an expected uniform spatial gradient in the beam's electrostatic repulsion forces. Third, it is shown that the offset-pole configuration also results in a modest tilt in the beam's major axis in the cross-sectional plane. For many applications, such tilt might well be negligible, but it may represent a disadvantage for situations in- volving high beam densities and very large beam aspect ratios (ratio of wide to narrow transverse dimensions). In comparison, the PCM-PQM hybrid magnetic focusing configuration provides equally impressive focusing capability (for high-space-charge beams), but has the extra advantage of independent control over the magnetic field parameters determining narrow and wide transverse dimension focusing. As a result, as a practical matter, attainment of matched beam equilibria appears to be far less problematic. In addition, the PCM-PQM hybrid focusing configuration is free of any adverse beam tilt effects. The offset-pole configuration still retains one advantage over the PCM-PQM configuration: open access via the sides of the beam, which may be attractive to some applications requiring injection or extraction of $\mathrm{rf}$ or microwave radiation.

\section{ACKNOWLEDGMENTS}

The authors acknowledge support for this work by the DoD Tri-Service Vacuum Electronics Initiative as managed by AFOSR; by ONR funding, administrated by the Naval Research Laboratory; and by a NSF Presidential Young Investigator Award. Computational support for the simulations was provided, in part, by the University of Wisconsin College of Engineering. Use of the MAGIC PIC code was supported by the MAGIC User's Group, with much appreciated problem specific advice and support by Dr. Ludeking and Dr. Smithe. Many helpful suggestions and discussions with Dr. J. Scharer, Dr. R. True, and Dr. C. Armstrong, as well as L. Lazaar are gratefully acknowledged.

${ }^{1}$ O. Buneman, J. Electron. Control 3, 507 (1957).

${ }^{2}$ J. S. Cook, R. Kompfner, and W. H. Yocom, Proc. IRE 45, 1517 (1957).

${ }^{3}$ P. A. Sturrock, J. Electron. Control 7, 162 (1959).

${ }^{4}$ P. A. Sturrock, J. Electron. Control 7, 153 (1959).

${ }^{5}$ H. F. Webster, J. Appl. Phys. 26, 1386 (1955).

${ }^{6}$ R. L. Khyl and H. F. Webster, Proc. IRE 3, 172 (1956).

${ }^{7}$ C. C. Cutler, J. Appl. Phys. 27, 1028 (1956).

${ }^{8}$ J. R. Pierce, IEEE Trans. Electron Devices 3, 183 (1956).

${ }^{9}$ W. Knauer, J. Appl. Phys. 37, 602 (1966).

${ }^{10}$ O. Buneman, R. H. Levy, and L. M. Linson, J. Appl. Phys. 37, 3203 (1966).

${ }^{11}$ T. M. Antonsen and E. Ott, Phys. Fluids 18, 1197 (1975).

${ }^{12}$ R. Adler, O. M. Kromhout, and P. A. Clavier, Proc. IRE 43, 82 (1955).

${ }^{13}$ H. S. Uhm, S. Ben-menahem, and D. Yu, Phys. Plasmas 1, 3686 (1994).

${ }^{14}$ C. A. Kapetanakos, D. A. Hammer, C. D. Striffler, and R. C. Davidson, Phys. Rev. Lett. 30, 1303 (1973).

${ }^{15}$ J. H. Booske, W. W. Destler, Z. Segalov, D. J. Radack, E. T. Rosenbury, J. Rodgers, T. M. Antonsen, Jr., V. L. Granatstein, and I. D. Mayergoyz, J. Appl. Phys. 64, 6 (1988).

${ }^{16}$ D. U. Yu, J. S. Kim, and P. B. Wilson, in Proceedings of Advance Accelerator Workshop, Port Jefferson, NY, 1992, pp. 85-102.

${ }^{17}$ K. R. Eppley, W. B. Herrmansfeldt, and R. H. Miller, in Proceedings of the 1987 IEEE Particle Accelerator Conference, IEEE Catalog No. 87CH2387-9, Vol. 3, pp. 1809-1811 (1987).

${ }^{18}$ J. H. Booske, B. D. McVey, and T. M. Antonsen, Jr., J. Appl. Phys. 73, 4140 (1993).

${ }^{19}$ J. H. Booske, A. H. Kumbasar, and M. A. Basten, Phys. Rev. Lett. 71, 3979 (1993).

${ }^{20}$ J. H. Booske, M. A. Basten, A. H. Kumbasar, T. M. Antonsen, S. W. Bidwell, Y. Carmel, W. W. Destler, V. L. Granatstein, and D. J. Radack, Phys. Plasmas 1, 1714 (1994).

${ }^{21}$ J. T. Mendel, C. F. Quate, and W. H. Yocom, Proc. IRE 42, 800 (1954).

${ }^{22}$ M. A. Basten, Ph.D. dissertation, University of Wisconsin-Madison, 1996.

${ }^{23}$ R. True, IEEE Trans. Electron Devices ED-31, 353 (1984). 
${ }^{24}$ Z.-X. Zhang, V. L. Granatstein, W. W. Destler, S. W. Bidwell, J. Rodgers, S. Cheng, T. M. Antonsen, Jr., B. Levush, and D. J. Radack, IEEE Trans. Plasma Sci. PS-21, 760 (1993).

${ }^{25}$ TOSCA code, Vector Fields Limited, Oxford, UK.

${ }^{26}$ PANDIRA code. Poisson/Superfish Code Group. Los Alamos Accelerator
Code Group, report LA-UR-87-1263.

${ }^{27}$ B. Goplen, L. Ludeking, D. Smithe, and G. Warren, Comput. Phys. Commun. 87, 54 (1995).

${ }^{28}$ G. Caryotakis, IEEE Trans. Plasma Sci. 22, 683 (1994).

${ }^{29}$ T. Wessel-Berg, Tech. Dig. Int. Electron Devices Meet. 93, 153 (1993). 